\title{
THE FORMATION OF COLLABORATIVE CHAINS FOR CONCEPTUAL DESIGN
}

\author{
Antonio P. Volpentesta, Maurizio Muzzupappa \\ Universitàdella Calabria, volpentesta@deis.unical.it,muzzupappa@unical.it
}

ITALY

\begin{abstract}
In this paper, we propose a collaborative and joint approach for the identification of partners and the formation of virtual enterprise for concept designing, without compromising design rights or proprietary knowledge. Besides, we introduce a framework for a Multi-Agent System model which is capable of supporting such an approach. The approach described in this paper enables the formalization of distributed processes which lead to the emergence of both conceptual design chains and functional architectures of an innovative product.
\end{abstract}

\section{INTRODUCTION}

Previously proposed Virtual Enterprise (VE) models in advanced manufacturing are generally oriented to the management of initial phases of a product lifecycle, but there is also an increasing interest in the exploration of the management processes of a VE that is specifically devoted to the generation a product concept in the initial design phase.

The current tendency for gradual moves towards collaborative commerce increasingly leads one to study VEs which can efficiently operate in an e-design market involving more complex exchanges, i.e. not reducible to buy/sell transactions. For these VEs, a more effective conceptual design phase incorporating the management of a wider range of competencies and knowledge becomes crucial to achieve new and innovative results.

Conceptual Design refers to the creative phase of the design process which, according to authoritative contributions to the established literature (Ulrich, 1995, Rodgers, 2001), is placed between the product planning phase and the embodiment design phase. In other words, it includes all logical processes starting from the task clarification phase, which lead the designer or the project team, to formulate hypotheses, and search for solutions, and thus define the product architecture during the ideation phase. The scenario we focus on is based on the assumption that an enterprise has identified, in a knowledge market place, specific opportunities which can be addressed in the formulation of a product concept. In this case, new decision problems arise in VE management processes, particularly in the selection of partners and the identification of the organizational-informational structure. They should deal with the management of distributed knowledge management in an entire network of Design Offices and which cannot be tackled separately and sequentially, as happens 
when the classical life cycle of a virtual enterprise is adopted. As matter of fact, this life cycle comprises the identification, formation, operation and termination phases and the decision processes are sequentially related (Strader, 1998). Its adoption seems to be appropriate when the VE goal is essentially to deliver more timely and qualitative services and products (Weigand, 2002). It is less effective in a knowledge market (Davenport, 1998), i.e. where the VE business goal is basically to sell knowledge-professional services or knowledge products (i.e. innovative product concepts) and possible interactions between VE members are knowledge based transactions.

In particular, in a concept design market, the classical sequential approach to the identification and formation phases in the VE life cycle cannot be applied for the following reasons:

- a full description of the required product concept cannot be provided in advance (as it assumed in the identification decision process);

- enterprise rarely give away valuable concept design knowledge without expecting something in return. The formation process should utilize only information that is strictly needed to define a VE architecture (members, their interactions and their roles in contributing toward the VE goal, namely, the generation of a new concept), but it could not rely on complete product part concepts description without compromising enterprise private knowledge.

To satisfy these new challenges we propose:

- a multi-agent system model for a concept design knowledge e-market;

- a collaborative and joint approach for the identification of partners and VE formation, without compromising design rights or proprietary knowledge.

This work has allowed the authors to develop in a companion paper a formal definition of logical structures and processes which arise in the formation of VEs in a Conceptual Design Knowledge e-market (Volpentesta, 2005).

\section{CONCEPTUAL DESIGN KNOWLEDGE E-MARKET}

We assume that a product is not yet available on the market, cannot be produced by assembling a different set of parts during production, and cannot be obtained by the addition of some application engineering to a basic design. In other words, to achieve a better response to such business opportunities, the enterprise needs to develop an innovative product concept by exploring the product functions and properties with regards to consumer preferences. This enterprise decides to rely on a Conceptual Design Knowledge market, where knowledge circulation enables the blending of the collaborative contributions of a set of enterprise partners, in order to form a VE which is, in principle, capable of bringing new product concepts to life. Extending the Yamamoto (2001) notion of knowledge market, we assume that a Conceptual Design Knowledge market consists of a set of Design Offices (DO) which supply Requests for Concept (RFCs), conditioned undertakings to respond to an RFC, and responses to RFCs. An RFC defines a set of requirements for a functional concept, by specifying characteristic parameters and their value range. These parameters generally refer to technical/functional requirements of a product element or part. However, they may also refer to requirements that a DO must satisfy when responding to the RFC, or to assessment criteria that may be used for 
ranking the concepts received from respondents to the RFC. Conditioned undertaking to respond to an $\mathrm{RFC}$ is an expression of interest to respond conditional on obtaining responses to other related RFCs. An answer to an RFC consists of a functional concept description including, if necessary, other additional useful details. Moreover, we hypothesize that the execution of VEs formation processes may be supported by a technological infrastructure with some self-organization abilities, and for this reason we use the term "e-market". An e-market can be seen as a virtual marketplace where geographically distributed business participants possibly unknown to each other advanced can meet and cooperate to the purpose of achieving a common business goal. In our case, business participants are DOs interlinked by a network infrastructure and the goal is the formation of a VE with the capacity to generate an innovative design solution.

\section{THE APPROACH}

In our approach, two main distributed processes should be sequentially executed in order to identify a set of VEs each of which is potentially able to generate the required product concept, with distinctive properties and functional features. A further characteristic is that the product's functional architecture generated by a VE is isomorphic to the logical structure of the VE itself (see figure 1).

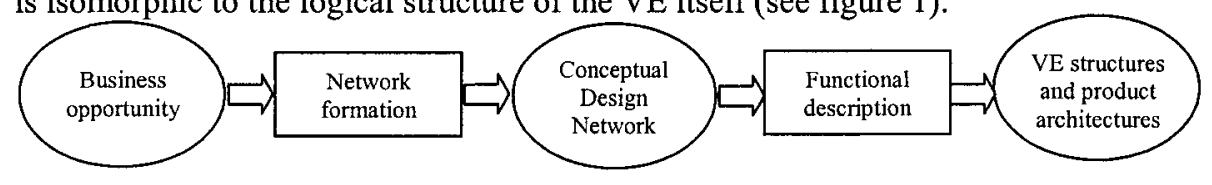

Figure 1. Objects and processes in our approach

The first process starts as soon as a DO, participating in the e-market, launches an initial RFC.

Conceptual Design Network formation. In this process each DO may carry out a task which leads to conditional undertaking to respond to an RFC: once the DO has viewed RFC, it carries out an exploratory consideration of functional features to decide should respond to the RFC. In such a case, it may issue related RFCs to the e-market and will respond to the initial RFC only if the related RFCs generate appropriate and sufficient responses to enable it to do so; contextually, it expresses its undertaking to respond to the $\mathrm{RFC}$, provided that it can receive the required responses to its RFCs. Multiple executions of this task, carried out by different DOs, induce a recursive formation of a Design Request Network which is a set of relations between RFCs, expressions of interest and DOs. Finally, the process extracts the Conceptual Design Network from the Design Request Network. The Conceptual Design Network consists of all logical structures of a VE which, relying on a collaborative and minimal organization, are potentially capable of responding to the given RFC coming from the e-market. On the one hand, the logical structure of a VE defines a decomposition of the initial RFC in other less complex ones, on the other hand it identifies the DOs and the requirements of their knowledge exchanges and their collaboration in order to respond to the given RFC.

Product Concept Functional Descriptions. Such a process is a recursive composition of Functional Description Tasks carried out by DOs along the 
Conceptual Design Network. A Functional Description Task consists of a more or less detailed description of a new product or a specific sub-system in accordance with requirements set out in RFC to which the DO is responding. It is important to underline that the execution of this task does not necessarily involve designing sharing. The task could be carried out by a DO on the basis of both the organizational information of the VE which the DO seeks to join and the condition of the information used in the entire Functional Description process occurring along the VE structure. Of course the task can start only if the DO has received all the required RFCs responses.

At the end of process, all possible architectures of VEs, capable of generating an innovative product concept, are obtained. Moreover, the DO issuing the initial RFC has access to all the responses. Only in a subsequent phase, VE members are required to exchange design and useful technical details to get a fully description of the product concept (Volpentesta, 2004). Such separation between the functional description phase and the one concerning full design definition, guarantees an adequate security level in the interaction between DOs, i.e. an interaction that allows sharing only of the information which is required for a functional description of a product concept, without compromising design rights and DO proprietary knowledge. Furthermore, technical information exchange is always restricted between two DOs, so that no DO can get a complete knowledge of all the functional features and so obtain a competitive advantage over other DOs. Of course, once a $\mathrm{VE}$ is established, a negotiation protocol has to be defined to initiate the operating phase in the VE life cycle.

\section{THE MULTI AGENT SYSTEM MODEL}

During the past decade, MAS modelling has emerged as promising discipline and many MAS have been proposed in distributed manufacturing (Hao, 2005). In conceptual design, the usage of a MAS, which fully automates the formation of collaborative chains, seems to be very challenging. Our proposal has more limited goals: the MAS should be intended only as a tool which reduces administrative time spent by a DO on managing RFCs, expressions of interest and RFCs responses and which allows the market broker to offer a set of a e-services for the formation of a VE. In what follows, we sketch a Multi-Agent System model in order to support the above mentioned processes that lead to the establishment of VEs oriented to the generation of an innovative product concept in the Conceptual Design Knowledge emarket. The general architecture of such a system includes several heterogeneous and semi-autonomous agents representing the several independent DOs, and also a special agent, called a Market Broker Agent, that assists the VE's formation processes. In our proposed system, only managerial information is exchanged between DO agents and the Market Broker agent, while technical information, i.e. RFC responses, may be exchanged only between two DO agents.

\section{$\checkmark \quad$ Market Broker Agent}

When a specific opportunity is identified, a DO activates a Market Broker Agent in the e-market system and sends an initial RFC to it. This agent plays the role of coordinator in the e-market where it is placed and its main goal is the VE's 
formation. Its structure includes three principal modules "RFC Management", "Design Structure Formation" and "Functional Description Process Control".

The RFC Management module contains a database of DOs participating in the emarket. Once a new RFC has been received from some member of the market the module forwards it to all DOs. Besides it archives expressions of interest from DOs submitting conditional undertakings to respond to certain RFCs. By analysing data collected in the RFC Management module, the Design Structure Formation module builds the Design Request Network, representing the relationship between RFCs, expressions of interest and DOs. More importantly, such a module is responsible for:

- extracting the Conceptual Design Network, which underlies an organizational model for the development of the collaborative/competitive conceptual design,

- identifying all VE logical structures, which are potentially able to collaboratively generate the required product concept,

- transmitting information to a DO agent about other DOs that are potentially eligible to participate in the formation of VE. In particular, it informs a DO of the partners that are required to exchange RFC responses with it.

The last module is devoted to gather data about Functional Description Tasks carried out by each DO in the Conceptual Design Network. In such a way, the Market Broker Agent is able to control the behaviour of the Functional Description Processes that are carried out somewhere along the VE structure. In particular, at any time, this module is able to determine the state or condition (on going, aborted or completed) of each Functional Description Process and to communicate it to all DOs involved in the related VE that is being formed.

\section{$\checkmark$ Design Office Agent}

A DO agent structure comprises different functional modules: "RFC Handler", "DO Knowledge", "VE Knowledge" and "Response Handler".

The RFC Handler receives RFCs from the Broker. Once the DO has looked over an RFC, say $r$, it carries out a functional features exploration, on the basis of its experience and skill, and decides whether it will respond to it. In such case, the DO may formulate some other RFCs, say $r_{1}, \ldots, r_{k}$, and forwards them the Broker. The $\mathrm{RFC}$ handler module sends to the Broker a message of expression of DO's interest to respond to $\mathrm{r}$; such a message should be interpreted as conditional undertaking to respond to $\mathrm{r}$ : the $\mathrm{DO}$ could respond to $\mathrm{r}$, provided it receives the responses $\mathrm{r}_{\mathrm{l}}, \ldots, \mathrm{r}_{\mathrm{k}}$. The VE Knowledge includes information, coming from the Broker about the formation process of each VE which the DO could belong to:

- Profiles of potential partners and their commitments;

- State of the Functional Description Process which is carried out by VE's potential partners

The DO Knowledge contains information about the agent itself (the selected RFCs which the DO has expressed an interest to respond to, the RFCs forwarded to the Broker, the RFCs responses coming from Response Handler module of the agent itself, the state of the functional description tasks which are carried out by the DO itself). The Response Handler receives from other DO Agents responses to the RFCs previously forwarded by the DO. Once the DO has carried out a Functional Description Task related to RFC such a module is capable of sending the RFC answer to DO Agents which have required it and, contextually, a message notification to the Broker (see figure 2). 


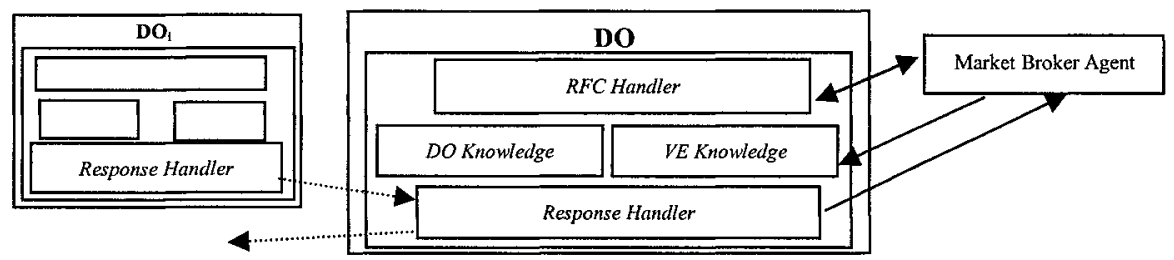

Figure 2. The DO Agent structure.

\section{EXAMPLE SCENARIO}

A certain enterprise has identified a specific opportunity which can be addressed by the design of an innovative vehicle to be used exclusively in shopping centres, airports or campuses. This enterprise $\left(\mathrm{DO}_{0}\right)$ activates the Market Broker Agent that forwards the initial RFCo (see table 1) to the market. Different Design Offices give conditional undertakings to respond to this $\mathrm{RFC}$. The $\mathrm{DO}_{1}$ proposes a concept consisting of a three wheel vehicle with a compressed-air engine of small dimensions, a seat for the passenger and a rack for bags. The $\mathrm{DO}_{1}$ proposes to develop innovations in the product's style, in the stacking procedures and in the recharge system of the parked vehicles. To achieve those proposals, the $\mathrm{DO}_{1}$ requires the collaboration of other DOs and decides to communicate a set of RFCs to the Market Broker Agent (see table1). The Market Broker Agent forwards these requests the market and will communicate to $\mathrm{DO}_{1}$ any resultant expressions of interest in these RFCs. A second $\mathrm{DO}_{2}$ plans to design a four-wheel vehicle with an intelligent driving system located under the pavement of the place of operation: this forces the vehicle to move only over an invisible "path". The vehicle is equipped with a laser which signals the presence of obstacles on the road in order to stop it. Since the $\mathrm{DO}_{2}$ does not have the know-how in an appropriate electronic control system, it searches for collaboration on the market and forwards appropriate RFCs to the Market Broker Agent (see tab.1). $\mathrm{DO}_{3}$ proposes to design an innovative trolley-like vehicle which can covert to an electrical three wheel vehicle. $\mathrm{DO}_{3}$ is not able to design a transmission system and wishes to make use of skills available on the market to respond to the initial RFC.

Table $1-\mathrm{RFC}_{0}$ and proposed concepts

\begin{tabular}{|c|c|c|c|}
\hline $\mathrm{RFC}_{\text {s }}$ by $\mathrm{DO}_{\mathrm{v}}$ & I concept by $\mathrm{DO}_{\mathrm{B}}$ & II concept by $\mathrm{DO}_{2}$ & III concept by $\mathrm{DO}_{3}$ \\
\hline \multirow[t]{4}{*}{$\begin{array}{l}\text { design of an innovative vehicle: } \\
\text { - avaliable for anyone with the } \\
\text { place of operation, } \\
\text { - very easy to drive, } \\
\text { - easily stored and re-charged } \\
\text { when not in use. }\end{array}$} & (3) & & 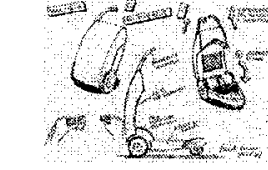 \\
\hline & $\begin{array}{l}\mathrm{RFC}_{1} \text { : request to design an } \\
\text { electronic control system }\end{array}$ & $\begin{array}{l}\mathrm{RFC}_{4} \text { : request to design a } \\
\text { laser system for automatic } \\
\text { obstacle recognition }\end{array}$ & $\begin{array}{l}\mathrm{RFC}_{6} \text { : request to design a } \\
\text { transmission system }\end{array}$ \\
\hline & $\begin{array}{l}\mathrm{RFC}_{2} \text { : request to design a } \\
\text { plastic mould }\end{array}$ & $\begin{array}{l}\mathrm{RFC}_{5} \text {; request to design a } \\
\text { steel framework }\end{array}$ & \\
\hline & $\begin{array}{l}\mathrm{RFC}_{3} \text {; request to design a } \\
\text { compressed air engine }\end{array}$ & & \\
\hline
\end{tabular}


The formation process of the Design Request Network starts with the launch of $\mathrm{RFC}_{0}$. Figure 3 represents the state of the network after some DOs have given conditional undertakings to respond to $\mathrm{RFC}_{0}$. The notation (RFCo :- $\mathrm{RFC}_{4} \mathrm{RFC}_{5}$ ) indicates that, in order to respond to RFCo, answers to $\mathrm{RFC}_{4}$ and $\mathrm{RFC}_{5}$ are necessary. The other notations are interpreted in a similar manner.

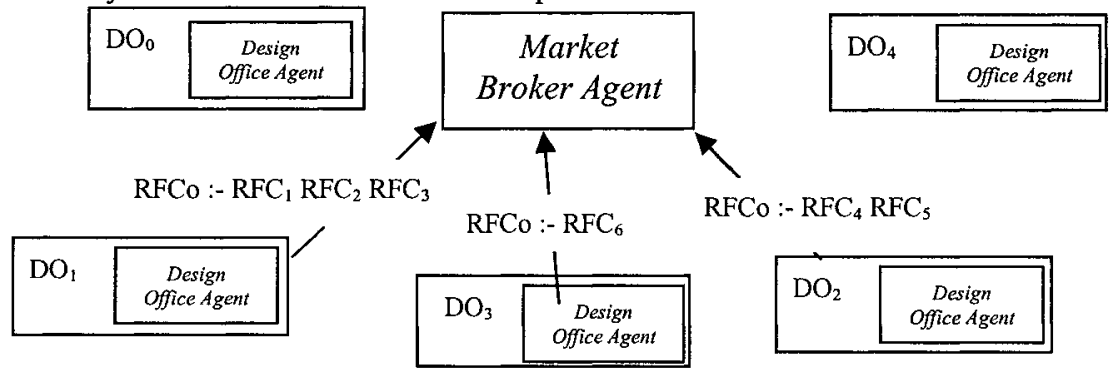

Figure 3. Expressions of interest.

At the end of this recursive process, a Design Request Network is formed. It is possible to use a hypergraph to represent this logical structure. In this hypergraph, a node is associated with RFC, while a hyperarc is associated with a DO that has expressed interest in responding to the RFC associated with its head. In the hypergraph, there is a special node associated with a $\mathrm{RFC}_{\text {dummy }}$ for which no answer is required. In figure 4, we have represented a particular Design Request Network in which no DO expresses an interest in the $\mathrm{RFC}_{3}$ (request of a compressed air engine). It follows that the first $\mathrm{DO}$ will not be able to produce a solution to $\mathrm{RFC}_{0}$, notwithstanding an expression of interest to do so.

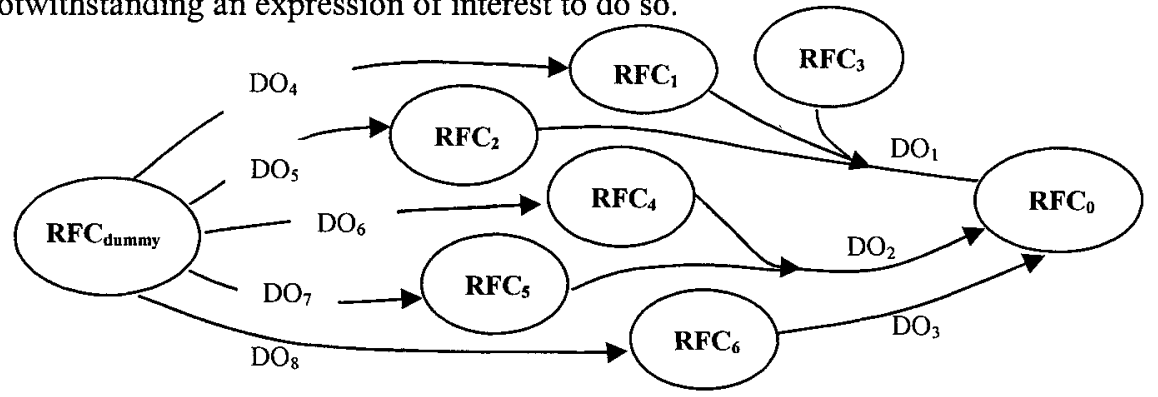

Figure 4. The Design Request Network

The definition of this hypergraph is essential to the extraction of all logical structures of Virtual Enterprises that are able to generate a new concept to respond to the initial RFC. At the end of the process, the Market Broker Agent has also collected all the information about substructures of VE which cannot be successfully completed. Figure 5 shows clearly how $\mathrm{DO}_{2}-\mathrm{DO}_{6}-\mathrm{DO}_{7}$ and $\mathrm{DO}_{3}-\mathrm{DO}_{8}$ can form two VEs capable of producing a concept responding to the initial RFC. This example has shown how structured aggregations of distributed design offices may emerge in a conceptual design market. Such aggregations are eligible candidates to form a VE. Starting from this point, the phase concerned with Product Concept Functional Descriptions should be executed. Only those aggregations which succeed 
after this phase would be able to activate managerial procedures aimed at forming a VE.

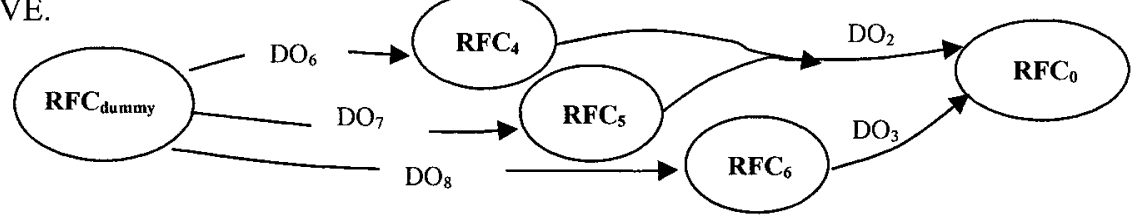

Figure 5. The Conceptual Design Network

\section{CONCLUSIONS AND FUTURE WORK}

We have presented a collaborative approach and a support MAS for the formation of a VE in a Conceptual Design Knowledge e-market. By adopting such an approach, the functionality of a new product and the structure of a VE which is capable of designing it, take shape in a circular way. In particular, we have focused on collaborative and distributed analysis of the first phases of the design activity which lead, on the one hand, to the identification of DOs and informational exchanges which take place between them, and, on the other hand, to the definition of possible functional architectures for the product. A fundamental feature of the proposed approach is the guarantee of an adequate level of mediation security among the DOs involved in the processes which we have considered to occur in the Conceptual Design Knowledge e-market. Finally, it is important to point out that the results of the present study may be used for further investigations in order to:

- give a complete formal definition of logical structures and processes which arise in the formation of VEs in a Conceptual Design Knowledge e-market;

- indicate a feasible implementation of the proposed MAS architecture, on the basis of emerging technologies, such as business objects and peer to peer; this problem is strongly felt by researchers and software producers who are involved in collaborative commerce along a Design Chain (Dhbrown.com, Ptc.com,...);

- define economic models and negotiation protocols for Design Chain management in a design phase, successive to the phase treated in this paper.

\section{REFERENCES}

1. Davenport T., Prusak, L., Working Knowledge, (Harvard Business School Press, 1998).

2. Hao Q., Shen W. Wang L., Towards a Cooperative Distributed Manufacturing Management Framework, Computers in Industry, vol. 56, pp.71-84, (2005).

3. Rodgers P.A., et al., The Management of Concept Design Knowledge in modern product development organizations, Int. Journal of Computer Integrated manufacturing, vol. 14, n.1, pp.108-115, (2001).

4. Strader T. J., Lin F., Shaw M.J., Information Infrastructure for Electronic Virtual Organization Management, Decision Support System, vol. 23, pp. 75-94, (1998).

5. Ulrich K., Eppinger S., Product Design and Development, (McGraw-Hill, New York, 1995).

6. Volpentesta A., Muzzupappa M., N. Frega, S. Rizzuti, Product Design Data Modelling for Review Process Management, J. of Engineering Design, vol.15, n.1, pp.53-67 (Taylor\&Francis Ltd, 2004).

7. Volpentesta A., Muzzupappa M., Identifying Partners and Logical Structures for VEs Oriented to Conceptual Design, submitted for publication.

8. Weigand H., Van den Heuvel W.J., Cross-Organizational workflow integration using contracts, Decision Support System, vol. 33, pp. 247-265, (2002).

9. Yamamoto H., Ohta T., Development of a knowledge market based on reputation, and absorption of uncertainty in electronic commerce, Proc. of the 5th World Multi-Conference on Systemics, Cybernetics and Informatics, vol.8, pp. 394-399, (2001). 